\title{
Extensible Hardware Architecture for Mobile Robots
}

\author{
Eric Park*, Linda Kobayashi, and Susan Y. Lee* \\ Intelligent Robotics Group \\ NASA Ames Research Center \\ Moffett Field, CA 94035-1000, USA \\ \{epark,lkobayashi,sylee\}@arc.nasa.gov
}

\begin{abstract}
The Intelligent Robotics Group at NASA Ames Research Center has developed a new mobile robot hardware architecture designed for extensibility and reconfigurability. Currently implemented on the $K \boldsymbol{g}$ rover, and soon to be integrated onto the KlO series of human-robot collaboration research robots, this achitecture allows for rapid changes in instrumentation configuration and provides a high degree of modularity through a synergistic mix of off-the-shelf and custom designed components, allowing eased transplantation into a wide variety of mobile robot platforms. A component level overview of this architecture is presented along with a description of the changes required for implementation on $K I 0$, followed by plans for future work.
\end{abstract}

Index Terms- modular, extensible, hardware architecture, mobile robot, $\mathrm{k} 9$

\section{INTRODUCTION}

Mobile robots used for research and development are in increasing demand as the application space for robotics widens. With this demand comes the need for a smarter way to produce reliable mobile robots with a proven set of avionics. Time and money lost in developing custom architectures for each new robot makes it increasingly important to develop extensible hardware architectures that can expand and accommodate rapid changes in configuration, including transfer to an entirely different mobile platform [1]. We have developed a new hardware architecture that makes optimized use of both off-the-shelf and custom designed hardware in order to achieve flexibility and extensibility. We believe this architecture is capable enough to be used as the basis for a wide variety of current and future mobile robot platforms.

\section{BACKGROUND}

The extensible hardware architecture described is currently implemented on the $K 9$ rover and is being transplanted to two $K 10$ robots with the goal of supporting research into peer-to-peer human-robot collaboration. These two new robots vary from $K 9$ both in instrumentation and capabilities. However, we aim to show that $K 9$ 's extensible architecture is scalable enough to meet the needs of K10 with minimal redesign.

\footnotetext{
* Contractor to NASA by QSS Group, Inc
}

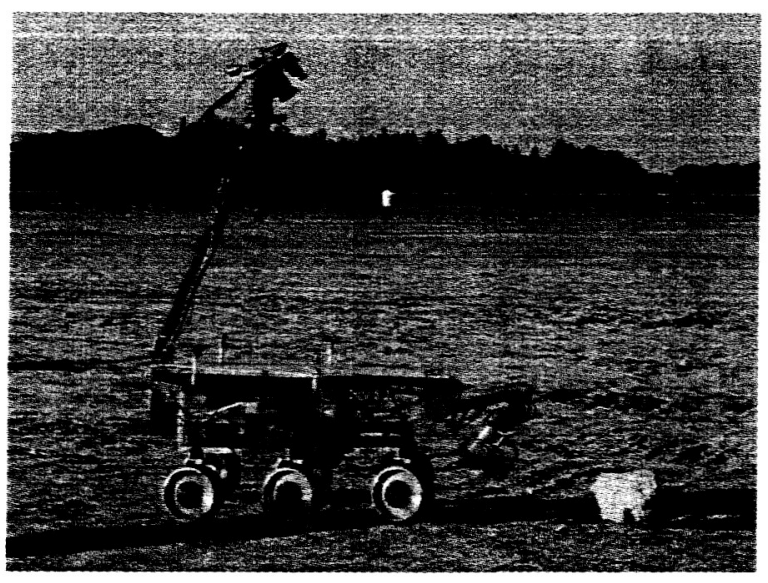

Fig. 1. $K 9$ rover

\section{K9 Overview}

The $K 9$ rover is a 6 -wheel steer, 6-wheel drive rockerbogey chassis outfitted with electronics and instruments appropriate for supporting research relevant to remote exploration [2] [3] [4]. K9's overall dimensions are $1.05 \mathrm{~m}$ long by $0.85 \mathrm{~m}$ wide by $1.6 \mathrm{~m}$ high, with a mass of approximately $65 \mathrm{~kg}$ and a top speed of approximately 6 $\mathrm{cm} / \mathrm{sec} . K 9$ 's instrumentation suite consists of a Honeywell HMR3000 compass/inclinometer, an Inertial Sciences inertial measurement unit (IMU), a Novatel differential global positioning unit (DGPS), a 5-DOF arm with a custom. microscopic imager, and three sets of stereo camera pairs, two of which are attached to a pan-tilt unit set atop a rigid mast.

K9's software architecture is based on Coupled Layered Architecture for Robotic Autonomy (CLARAty) [5] developed at Jet Propulsion Laboratory (JPL) in collaboration with NASA Ames Research Center (ARC) and Carnegie Mellon University. The use of CLARAty allows us to easily transplant our control software to other CLARAty based robots with no modification to high-level code.

\section{K10 Overview}

$K 10$ is our next generation mobile robot research platform to be used within the Intelligent Robotics Group (IRG) at ARC. This series of robots is designed to be 


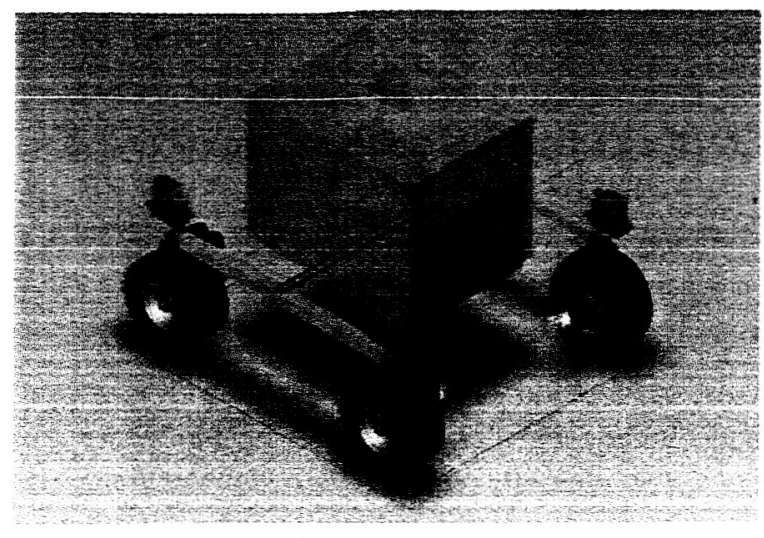

Fig. 2. Conceptual model of $K 1 O$ (credit: Thomas Hsiu)

a cost-efficient, easily maintainable robotic testbed using as many commercial off-the-shelf components as possible. Each robot features a 4-wheel steer, 4-wheel drive rocker chassis and a top speed of $30 \mathrm{~cm} / \mathrm{sec}$, comparable to human-walking speed. Hard points on all sides allow attachment of additional components including antennas, masts, arms, and other equipment.

$K 10$ 's avionics strives to duplicate those of $K 9$ wherever possible, including the computing infrastructure, power system, and instrumentation. Although the $K 10$ robots will draw significantly more power than $K 9$ (approx. $500 \mathrm{~W}$ versus $100 \mathrm{~W}$ nominal), K9's power architecture is extensible enough to accommodate K1O's power requirements with minimal modification, thereby saving engineering time and development costs.

\section{ARCHITECTURAL OVERVIEW}

K9's hardware architecture consists of several core avionics elements including a Li-Ion based distributed power system, integrated motion controllers, and a laptop based computing infrastructure.

\section{Power System}

$K 9$ 's power system has four key elements that allows for easy reconfiguration and expansion: a self-monitoring battery charger board, "smart" Li-Ion battery packs, a power distribution board, and several distributed power switching modules.

Battery Charger Board: K9's battery charging system consists of a single board capable of supporting the charging and monitoring of up to six hot-swappable battery packs. If necessary, several of these in-house designed boards can be stacked in series or parallel to accommodate additional batteries.

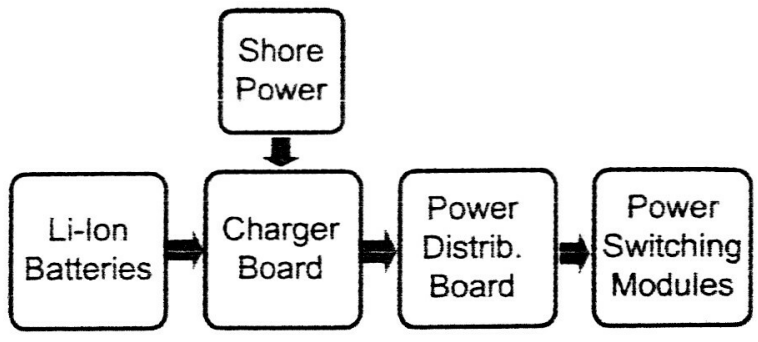

Fig. 3. Power System Block Diagram

The battery charging board uses an Atmel AVR ATMegal6L microcontroller and three Linear Technology LTC1960 Li-Ion charging chips. The presence of a microcontroller allows the board to self-monitor each battery and individually charge them as necessary when external power is applied. A RS232 serial interface is provided to allow the host $\mathrm{CPU}$ to query battery status, however no host intervention is necessary to enable or disable charging. The autonomous nature of the battery charger enables easy expansion by allowing multiple chargers to be present in a robot with no additional supervisory load on the host CPU.

In this case, a custom solution was chosen over a commercial off-the-shelf charger because we were unable to find a charger that met our needs. Our battery charging board's stackable and autonomous nature underscores our emphasis on scalability with future expansion in mind.

Li-Ion Battery Packs: $K 9$ carries six Inspired Energy NL2024A22 "smart" battery packs in parallel. Each Li-Ion battery nominally provides $14.4 \mathrm{~V}$ at $6.6 \mathrm{AH}$ and possesses a SMBus interface for easy querying of status and charge capacity. Internal storage of charge state allows the batteries to be hot-swappable, enabling the rover to run continuously without downtime for charging. However, even without hotswapping, $K 9$ can typically run for a full day on a single charge. KlO will use the same battery packs, but twice as many to accommodate the high current requirements of the drive motors.

Although we experiemented with in-house battery packs before settling on Inspired Energy's batteries, going with a commercial vendor meant that $K 9$ 's batteries would be readily available and easily obtainable for significantly less than the cost of making custom batteries.

Power Distribution Board: The power distribution board is an in-house designed circuit board used on both $K 9$ and K1O. Its sole purpose is to take the power provided by the battery charging boards and distribute it to the various on-board instruments. Flexibility is achieved through the use of commercial Vicor DC-DC converters. Power from the battery charging boards is funneled through several Vicor converters which regulate the voltage up or down as necessary before being distributed to instruments. Since 
these units are available in many different output voltages, it is trivial to mix and match converters as needed. Each power distribution board is capable of providing three output voltages but can be extended by stacking boards as necessary to provide additional output voltages or more power per voltage source.

Power Switching Modules: Power switching of devices is performed in a distributed manner using custom USBenabled power switching modules. As the number of devices increases, additional power switching modules can be added proportionally. The use of USB as the communications interface allows for a high degree of expandability both in bandwidth and number of supported devices.

On $K 9$, devices and instruments including the wheels. arm, pan-tilt, compass, and IMU are powered on and off as needed to conserve power when not in use. The power switching module (Figure 4 ) allows $K 9$ to selectively switch peripheral device power on and off via USB (USB 1.1 and USB 2.0 compatible).

Each power switching module consists of a DLP Design DLP-245PB microcontroller module, which allows a host computer to control several LO lines via USB, and an in-house designed daughterboard which mates with the microcontroller module to allow it to handle high current power switching. The actual power switching is performed by Infineon Technologies BTS428L2 MOSFET power switches connected to the DLP-245PB's VO lines. The daughterboard features three switched power lines, each of which can handle up to 3 amps continuous current and assume one of three input voltages. If a device has higher power requirements, multiple switched lines may be ganged together to achieve a higher effective current rating up to 9 amps. The daughterboard also measures the voltage and current on each switched power line using $A / D$ converters and current sense resistors. This information is communicated back to the host computer upon request in a manner similar to [6]. The sense resistor and current-sense amplifier is selected based on the power requirements of the associated device. On $K 9$, a typical setup consists of a maximum current rating of 2.5 amps and a maximum input voltage of about 20 volts per switched line.

The daughterboard is about 1.3 inches by 4.0 inches, only slightly larger than the microcontroller module itself, and has four mounting holes for easy attachment. Features including USB-based communication, small footprint, and configurable power requirements allows these boards to be easily integrated on any system having USB capability. The devices themselves appear as regular serial ports to the operating system. A CLARAty module with a simple API allows for easy control and monitoring of power consumption of each devices. Since a single USB port can control up to 127 devices and each power switching module can switch power for up to three devices, multiple power switching modules used in conjunction over USB

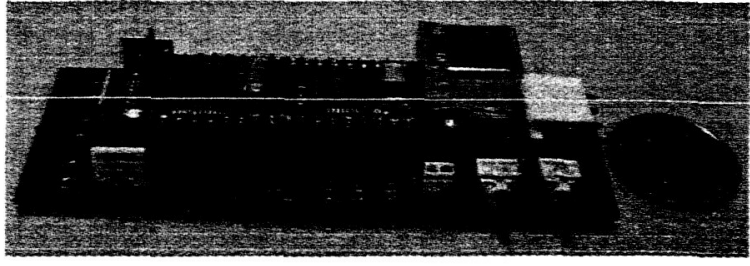

Fig. 4. USB-enabled Power Switching Module

affords a virtually unlimited number of devices to be switched. The addition of a new instrument or change in power requirements merely requires the addition of a new power switching module, or the changing of a few passive components respectively.

Both the power distribution board and the power switching modules achieve extensibility through a hybrid mix of off-the-shelf and custom components. Store-bought Vicors are paired with a custom designed PCB while the commercial DLP-245PB is complemented by an in-house designed daughterboard. In both cases, the two types of hardware are combined to create a module that greatly increases the extensibility of $K 9$ 's architecture.

Power Management: "Smart" batteries, fine-grain control and monitoring of power consumption, and automated charge management allows $K 9$ to make informed plans given resource constraints [7]. During autonomous operation, given a set of objectives and contingent plans, $K 9$ can choose to perform one set of activities over another set based on its current power consumption, remaining power, and each activity's power requirements. This ability also allows $K 9$ to maximize runtime by turning off unnecessary devices. Use of CLARAty and an extensible power system allows us to transfer this capability to $K 10$ with no reengineering of hardware or software.

\section{Integrated Motion Controller}

$K 9$ makes use of several six-axis motion controllers, designed at ARC and based on the JR KERR PIC-SERVO integrated motor control board design. Each motor control circuit employs a National LMD18200 H-bridge amplifier and the PIC-SERVO/PIC-ENC chipset. Each axis can drive up to 3 amps continuously ( 6 amps peak at $48 \mathrm{VDC} \max$ ) via pulse width modulation (PWM), accept encoder data, and perform trapezoidal and velocity profiling. Each sixaxis board communicates to the host over RS232 or as part of a RS485 bus, allowing 32 motors to be controlled from a single serial port. Either serial interface enables the motion controller boards to expand. By making use of off-the-shelf JR KERR PIC-SERVO motor control boards, 


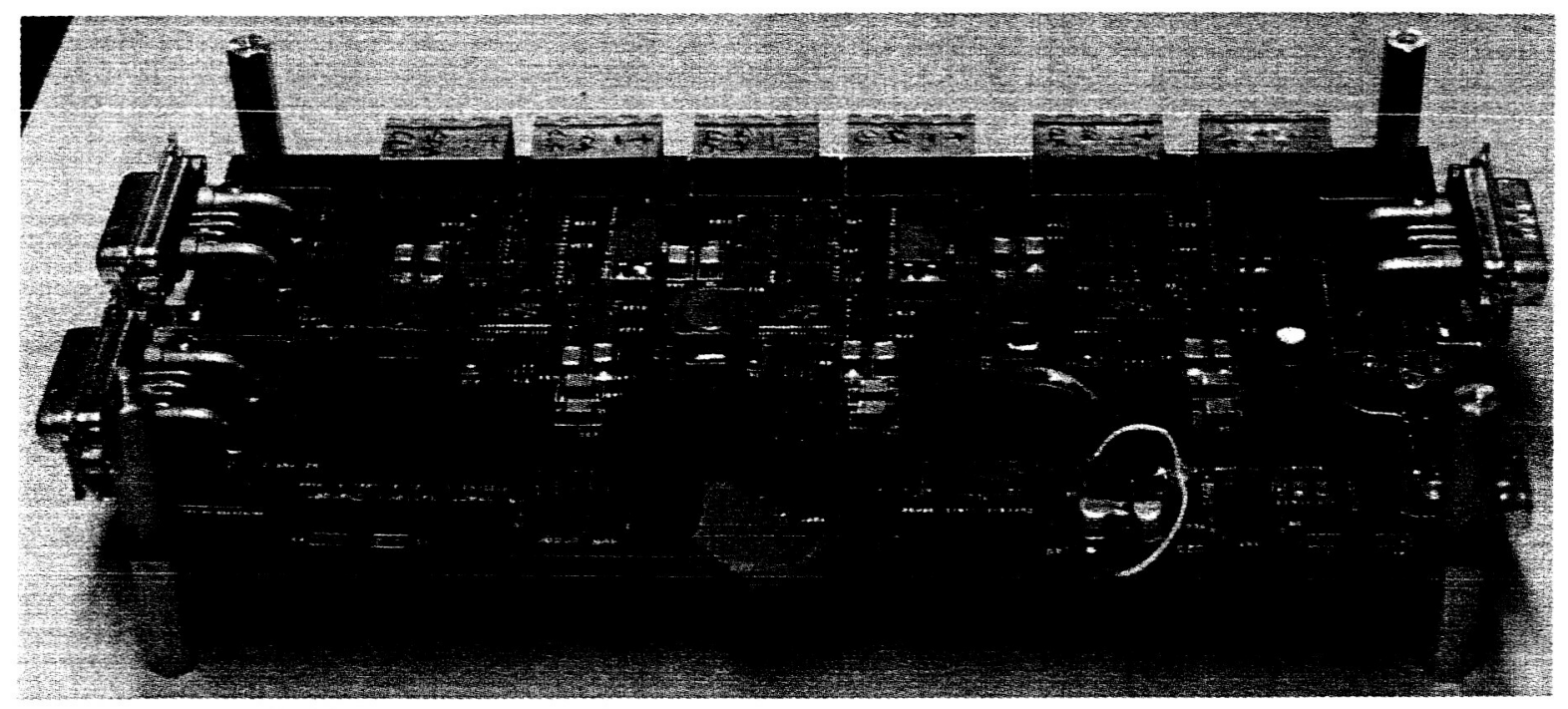

Fig. 5. Li-Ion Battery Charger Board

extensibility is restricted only by the number of RS485 devices allowed on a single chain. On $K 9$, four six-axis motion controller boards are used to drive six Maxon drive motors and six Maxon steer motors, a 5-DOF manipulator arm using MicroMo 1319 series motors, and a pan-tilt unit using two Pittman motors. Although initially designed solely to control the drive and steer motors, the board's flexible nature has allowed us to easily expand its use to meet $K 9$ 's changing motion requirements.

In order to achieve human-walking speeds, K10 will use Magmotor S15 brushed servo DC motors. These motors require significantly more power than the 3 amps a single LMD18200 can provide. Instead, KlO will use readily available IFI Robotics Victor 885 speed controllers made popular by the US FIRST and BattleBots robot competitions. K9's motion controllers provide an unamplified PWM output which can easily be fed into the PWM input on the Victor 885 's, allowing us to very easily accommodate any size motor with minimal effort.

\section{Computing Infrastructure}

Rapid advances in miniaturization and the development of high-speed serial communications protocols within the consumer computer industry have allowed us to forego $\mathrm{PC} / 104$ stacks and CompactPCI and replace them with powerful subnotebooks and hot-pluggable hubs, both of which allow effortless expansion and reconfiguration.

Host CPU: $K 9$ 's main CPU consists of an IBM Thinkpad X31 laptop with a $1.2 \mathrm{GHz}$ Pentium M processor. A mainstream laptop was chosen over an embedded solution for reliability, simplified debugging via the keyboard

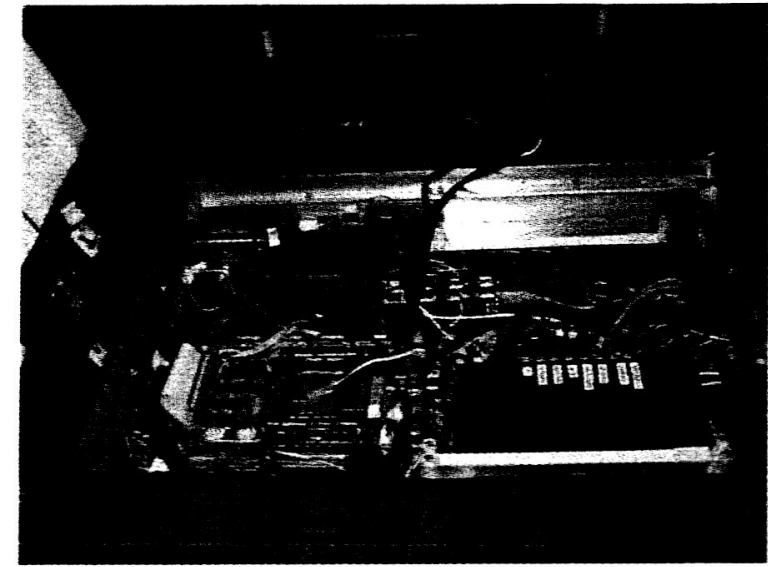

Fig. 6. $K 9$ Electronics Enclosure with Laptop Removed

and LCD, and easy replacement. There are only four cables connecting $K 9$ 's avionics to its host CPU: one USB cable, one Firewire cable, one Ethernet cable, and an $802.11 \mathrm{~g}$ RF cable. The USB, Firewire, and Ethernet cables all go into hubs within the electronics enclosure, while the RF cable goes to an $8 \mathrm{~dB} 2.4 \mathrm{GHz}$ antenna. Two Li-Ion laptop batteries provide over six hours of runtime while a PCMCIA $802.11 \mathrm{~g}$ card provides wireless connectivity.

$K 9$ currently runs Redhat Linux which was chosen for its large user base and compatibility with other applications. The use of a free and pervasive operating system provides us with a robust development environment, eased collaboration, and a large talent pool from which to draw. Linux's flexibility and scalability enables us to easily add, remove, and extend devices with minimal difficult. 
The robot's controller consists of a single multi-threaded application, which runs as a user executable. The use of distributed microcontrollers frees us from requiring a real-time operating system, thereby enabling us to dedicate our CPU to navigation, image processing, and other high-level tasks. Fine-grain motor control, power monitoring, and device management are offloaded to the motion control boards, battery charger board, and power switching modules respectively.

Communications: Communication across the various devices on $K 9$ is performed through a myriad of protocols including $\mathrm{i}^{2} \mathrm{c}$, SMBus, RS232, RS485, USB, Firewire, and Ethernet. Cables to the host CPU are minimized by employing only high-bandwidth hot-pluggable protocols: USB, Firewire, and Ethernet. The high speed interfaces in turn connect to hubs that branch off into a multiple low-bandwidth interfaces in a tiered topology. Firewire is used to interface to the three stereo camera pairs while Keyspan USB-serial adapters fan out from the USB hub to provide RS232 and RS485 interfaces for devices including the compass, IMU, DGPS, motion controller board, and battery charger board. The expandable and hot-pluggable nature of Firewire, USB, and Ethernet, along with Linux's support for such protocols allows for effortless reconfiguration and addition of devices. The addition of a new instrument simply involves connecting power to a power switching module, data to a serial interface, and telling the instrument's CLARAty module where to obtain the data.

\section{FUTURE WORK}

Over the last five years, $K 9$ 's avionics has continuously evolved to keep up with emerging technologies and changing requirements; $\mathrm{PC} / 104$ stacks were replaced with laptops, NiMH batteries and solar panels with high-capacity Li-Ion batteries, and NTSC cameras with high-resolution Firewire cameras. Given the complex nature of the system, issues-arise as the rover undergoes extensive use, in turn prompting us to find new solutions. The Keyspan USBserial adapter is a recent example of problematic hardware we have encountered. As we begin to transfer $K{ }^{9}$ 's avionics to $K 10$ we expect to come across more such issues, but with each evolution, we are confident the system as a whole will only continue to increase in stability and fexibility.

\section{Cyclades Serial Console Server}

After several months of use, it was discovered that the Keyspan USB-serial adapters do not scale well under heavy load. The Keyspan adapters would intermittently fail during bursts of data several hours into a run. In order to reduce downtime, a solution was proposed using a Cyclades TS800 console server. The TS- 800 provides eight RS232 serial ports and one Ethernet jack that allows socket,

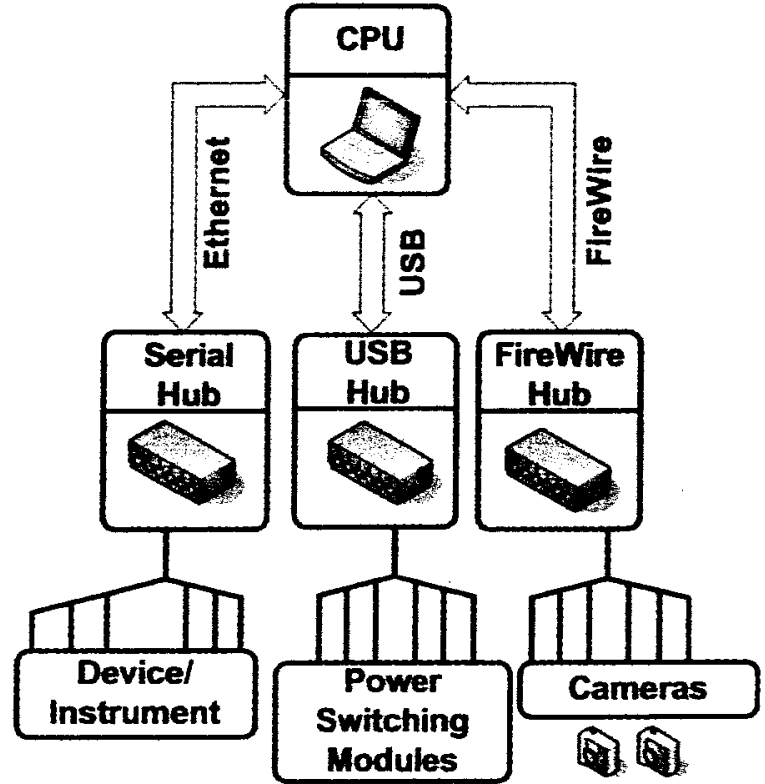

Fig. 7. System Diagram After Cyclades Integration

telnet, and ssh connections to the serial ports through the Ethernet interface. Similar to the USB architecture, the use of an Ethernet switch allows us to add an arbitrary number of serial ports. The TS- 800 contains a PowerPC processor and also runs Linux, thereby allowing us to easily make modifications and adjust parameters as necessary. In particular, in order to implement in-situ serial speed changes over a socket, a custom serial server was written and downloaded to flash memory. Integration of the TS-800 into $K 9$ is currently underway. $K 9^{\prime}$ 's post-Cyclades system diagram is given in Figure 7.

\section{KI0 Progress}

K10's design is currently being finalized with construction expected to begin shortly. Avionics integration will begin in October and we expect to have an operational research platform by the end of 2004 .

\section{CONCLUSION}

$K 9$ 's architecture features an optimized mix of off-theshelf and custom designed components, thereby taking advantage of the beneficial attributes of each. The lowcost and readily available volume of commercial components allows for easy cloning and scalability. In cases where commercial components are not available or do not meet our needs, custom designed components allow us to maintain our design objectives without compromising our ability to expand. The complementary nature of these two 


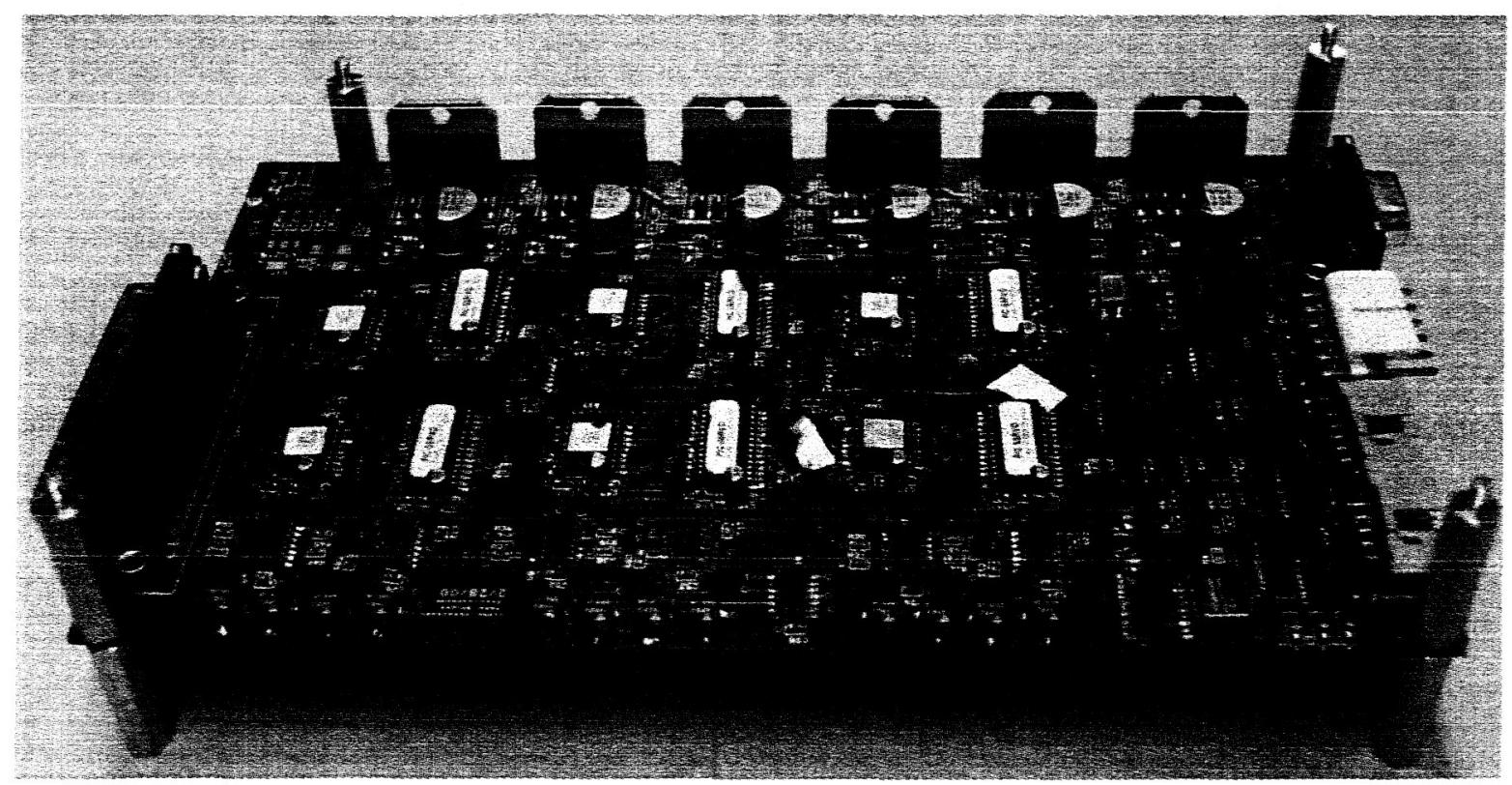

Fig. 8. Six-Axis Motion Controller Board

types of components is exemplified by our use of hybrid components consisting of both commercial and custom technologies.

By taking advantage of the synergistic nature of commercial and custom components, we have created an extensible hardware architecture that can be easily adapted and transferred betwen robot designs, thereby saving both time and effort while allowing the architecture to evolve and improve on each implementation.

\section{ACKNOWLEDGMENTS}

The authors of this paper would like to acknowledge the support of the Intelligent Systems and the Mars Technology programs. We would also like to acknowledge the support of other members of the Intelligent Robotics Group at NASA Ames Research Center: Clay Kunz, Anne Wright, Randy Sargent, Maria Bualat, and Illah Nourbakhsh.

\section{REFERENCES}

[1] S.G. Roh, K.H. Park, K.W. Yang, J.H. Park, H.S. Kim, H.G. Lee H.R. Choi.. "Development of Dynamically Reconfigurable Persona Robot," Robotics and Automation, 2004. Proceedings, ICRA '04. 2004 IEEE International Conference on. vol. 4. 26 April-1 May 2004 , pp. $4023-4028$

[2] L. Pedersen, R. Sargent, M. Bualat. C. Kunz, S. Lee, A. Wright "Instrument Deplovment for Mars Rovers." International Symposium on Artificial Intelligence. Robotics and Automation in Space. i-SAIRAS. 2003

[3] M. Matsuoka, S.M. Rock, M.G. Bualat, "Autonomous Deployment of a Self-Calibrating Pseudolite Array for Mars Rover Navigation," Position Location and Navigation Symposium. 2004. PLANS 2004 26-29 April 2004, pp. 733-739

[4] J. Bresina, M. Bualat, L. Edwards, R. Washington, A. Wright, "K9 Operations In May '00 Dual-Rover Field Experiment," Intemational Symposium on Artificial Intelligence. Robotics, and Automation in Space, i-SAIRAS, 2001

[5] R. Volpe, I. Nesnas, T. Estlin, D. Mutz, R. Petras, H. Das, "The CLARAty Architecture for Robotic Autonomy," Aerospaee Conference. 2001, IEEE Proceedings., vol. 1, pp. 121-132, iss., 2001

[6] Chung-Ping Young, M.J. Devaney, Shyh-Chyang Wang, "Univera Serial Bus Enhances Virtual Instrument-Based Distributed Power Monitoring," Instrumentation and Measurement, IEEE Transactions on. Vol. 50, Iss. 6. Dec 2001, pp.1692-1697

[7] R. Washington. K. Golden, J. Bresina, D.E. Smith, C. Anderson, T. Smith, "Autonomous Rovers for Mars Exploration," Aerospace Conference, 1999. Proceedings. 1999 IEEE, vol. 1, 6-13 March 1999, pp. $237-251$ 